Conclusions: Macrophage migration inhibitory factor and interleukin- 8 are highly produced in acute gout. Monosodium urate crystals induced macrophage migration inhibitory factor production in monocytes and interleukin-8 production in neutrophils with a reciprocal interaction between the two cytokines.

Acknowledgements: This work was supported by Konkuk University Medical Centre Research Grant 2016.

Disclosure of Interest: None declared

DOI: 10.1136/annrheumdis-2018-eular.2365

\section{AB0063 HIGH-EFFICIENCY TRANSDUCTION OF MESENCHYMAL STEM CELLS BY AAV2/DJ VECTOR FOR THEIR POTENTIAL USE IN AUTOIMMUNE DISEASES}

H. Zhang ${ }^{1}$, X. Tang ${ }^{1}$, C. Wang ${ }^{2}$, L. Sun ${ }^{1}$. ${ }^{1}$ Department of Rheumatology and Immunology, The Affiliated Drum Tower Hospital of Nanjing University Medical School, Nanjing, China; ${ }^{2}$ Viral Core, F.M. Kirby Neurobiology Center, Boston Children's Hospital, Boston, USA

Background: Mesenchymal stem cells (MSC), multipotential non-hematopoietic progenitors, can be isolated from various tissues and can modulate allogeneic immune cell responses. These properties make MSC as a promising potential treatment of autoimmune diseases. ${ }^{1}$ Our previous studies have found that bone marrow-derived (BM)-MSC from systemic lupus erythematosus (SLE) patients are defective structurally and functionally, ${ }^{2}$ treatment with modified and optimised MSC may bring a better effect on patients with autoimmune diseases. Most efforts have relied on adeno- and lentiviral vectors for delivering genes to MSC. Effective as these vectors may be, concerns regarding their immunogenicity and, in the case of lentivirus, the risk of insertional mutagenesis, have led to the pursuit of safer alternatives. Among these, adeno-associated virus (AAV) holds several advantages as a vector for human gene therapy. There are many serotypes of AAV available, and certain serotypes have been found to transduce specific cell types more efficiently than others.

Objectives: To determine the efficiency of different serotypes of AAV vectors for their ability to mediate transduction of different sources of MSC and assess whether AAV transduction affects MSC multipotentiality.

Methods: Serotypes 1, 2, 5, 6, 8, 9, PHP and DJ of AAV vectors were constructed in Viral Core, Boston Children's Hospital. The enhanced green fluorescent protein (eGFP) gene under transcriptional control of a CAG promoter was cloned into the AAV vector backbone. MSC derived from umbilical cord (UC), BM and amniotic fluid (AF) were isolated and approximately $1 \times 10^{5} \mathrm{MSC}$ were used for transductions with AAV vectors. eGFP expression was evaluated 3 days after transduction by fluorescence microscopy and flow cytometry. The capacity of MSC to differentiate in vitro was assessed.

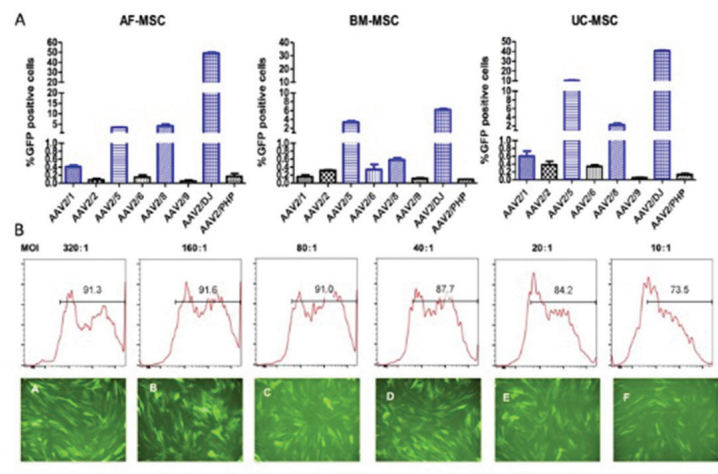

Figl A: the transduction efficiencies of AAVs (MOI=5:1) in AF-MSC, BM-MSC and UC-MSC, B: the transduction efficiencies of AAV/DJ in UC-MSC with different MOI.

Abstract AB0063 - Figure 1. A: the transduction efficiencies of AAVs (MOI=5:1) in AFMSC, BM-MSC and UC-MSC, B: the transduction efficiencies of AAV/DJ in UC-MSC with different MOI.

Results:

- AAV serotype DJ vector was the most efficient in transducing MSC. AAV was added directly to the medium at 5 multiplicities of infection (MOI), $41 \%$ UC-MSC was transduced by AAV2/DJ, while the transfection is $0.47 \%$, $0.3 \%, 10.5 \%, 0.3 \%, 1.84 \%, 0.06 \%, 0.16 \%$ by AAV2/1, AAV2/2, AAV2/5,
AAC2/6, AAV2/8, AAV2/9, AAV2/PHP (Fig 1A). Transduction efficiencies ranged from $73.5 \%$ for $\mathrm{MOI} 10 \%$ to $91.3 \%$ for MOI 320 in UC-MSC (Fig 1B).

- MSC derived from different tissues share a comparable level of transduction with the same AAV vector serotype. In our result, AAV2/DJ was the most efficient in transducing UC-MSC and AF-MSC

- AAV2/DJ transduced MSC retained the same multipotential activity to differentiate into osteogenic and adipogenic lineage as comparable to untransduced cells.

Conclusions: AAV2/DJ vector can be used as a highly efficient tool to modify MSC ex vivo for therapeutic transplantation for autoimmune diseases.

\section{REFERENCES:}

[1] Liang J, Wang D, Dominique F, Sun L. Mesenchymal stem cells for treating autoimmune diseases: The Chinese experience from lab to clinics. Curr Res Transl Med. 2016;64(2):115-20.

[2] Sun L, Akiyama K, Zhang H, et al. Mesenchymal stem cell transplantation reverses multiorgan dysfunction in systemic lupus erythematosus mice and humans. Stem Cells 2009;27(6):1421-32.

Disclosure of Interest: None declared

DOI: 10.1136/annrheumdis-2018-eular.5014

\section{AB0064 $\quad$ ROLE OF IL-35 IN THE REGULATION OF IMMUNE RESPONSE IN PATIENTS WITH RHEUMATOID ARTHRITIS}

I. Adamova ${ }^{1}$, M. Mravcova ${ }^{1}$, M. Vlcek ${ }^{1}$, A. Penesova ${ }^{1}$, K. Krskova ${ }^{1}$, Z. Radikova ${ }^{1}$ A. Havranova', R. Imrich ${ }^{1,2}$. ${ }^{1}$ Biomedical Research Center, Slovak Academy of Sciences, Bratislava; ${ }^{2}$ National Institute of Rheumatic Diseases, Piestany, Slovakia

Background: Interleukin 35 (IL-35) is a recently identified member of the IL-12 family of cytokines and represents a novel target for therapies of autoimmune, inflammatory, and infectious diseases, including rheumatoid arthritis (RA). ${ }^{\text {Choi et al. }}$ 2015

IL-35 is a heterodimer consisting of EBV-induced gene 3 (EBI3) and IL- $12 \alpha$ chain p35 (Nakano et al. 2015). In contrast to other IL-12 cytokine family members, IL35 appears to have anti-inflammatory and immunosuppressive properties mediated by induction of regulatory T and B cells; (Choi et al. 2015. Huang et al. 2017). In particular, IL-35 may play an important role in suppressing the inflammatory response by expanding regulatory $\mathrm{T}$ cells and in dampening the differentiation of Th17 cells (Niedbala et al. 2007).

Objectives: This study was designed to analyse effects of IL-35 on stimulated peripheral blood mononuclear cells (PBMC) and their subpopulations in RA patients and healthy controls.

Methods: PBMCs of 10 RA patients and 10 controls as well as CD14 +and CD4 +cells isolated from PBMCs using magnetic separation were cultured for 24 hours, and subjected to three conditions: no stimulation, stimulation with LPS (PBMC and CD14+) or stimulation with anti-CD3/anti-CD28 antibodies (PBMC and CD4+), and stimulation with added IL-35 (100 ng/ml). A panel of nine cytokines (IL-1 $\beta$, IL-6, IL-8, IL-10, IL-12 (p70), IL-17a, IFN- $\gamma$, MIP-1 $\beta$ and TNF) was analysed in the cell culture supernatants.

Results: RA patients had higher serum levels of IL-35 compared to healthy controls. A decreased secretion of IL-8 and increased secretion of TNF in the pres ence of IL-35 was observed in vitro in stimulated PBMCs of RA patients. In the control group, we observed an increased secretion of IL- 6 by PBMCs and decreased secretion of IL-10 by T lymphocytes as a result of IL-35 addition to stimulated cells.

Conclusions: In this study, we found elevated serum levels of IL-35 in RA patients suggesting a possible involvement of IL-35 in the pathogenesis of RA. However, in vitro, the effect of IL-35 on stimulated immune cells was partially antiinflammatory and partially pro-inflammatory, suggesting that the effect of IL-35 is pleiotropic and depends on the type and the state of the affected immune cell.

\section{REFERENCES:}

[1] Huang A, Cheng L, He M, et al. Interleukin-35 on B cell and T cell induction and regulation. J. Inflamm. (United Kingdom) 2017;14.

[2] Choi J, Leung PSC, Bowlus C, Gershwin ME. IL-35 and Autoimmunity: a Comprehensive Perspective. Clin Rev Allergy Immunol 2015;49:327-332. doi:10.1007/s12016-015-8468-9 
[3] Nakano S, Morimoto S, Suzuki S, et al. Immunoregulatory role of IL-35 in $\mathrm{T}$ cells of patients with rheumatoid arthritis. Rheumatology (Oxford) 2015;54:1498-1506. doi:10.1093/rheumatology/keu528

[4] Niedbala W, Wei XQ, Cai B, et al. IL-35 is a novel cytokine with therapeutic effects against collagen-induced arthritis through the expansion of regulatory $\mathrm{T}$ cells and suppression of Th17 cells. Eur $\mathrm{J}$ Immunol 2007;37:3021-3029. doi:10.1002/eji.200737810

Acknowledgements: Grant support: APVV-15-0228 and VEGA 2/0161/16

Disclosure of Interest: None declared

DOI: 10.1136/annrheumdis-2018-eular.6522

\section{AB0065 N-CADHERIN IS DOWN-REGULATED BY DECOY RECEPTOR 3 IN SPECIFICALLY RHEUMATOID SYNOVIAL FIBROBLASTS}

K. Fukuda ${ }^{1}$, Y. Miura ${ }^{2}$, S. Hayashi ${ }^{1}$, T. Maeda ${ }^{3}$, R. Kuroda ${ }^{1} .{ }^{1}$ Department of Orthopaedic Surgery, Kobe University Graduate School of Medicine, Kobe, Japan; ${ }^{2}$ Division of Orthopedic Science, Department of Rehabilitation Science, Kobe University Graduate School of Health Sciences, Kobe, Japan; ${ }^{3}$ Department of Immunology and Rheumatolog, Stanford University, Stanford, USA

Background: Decoy receptor 3 (DcR3) is a secreted decoy tumour necrosis factor receptor and competitively binds and inhibits the TNF family including Fasligand, LIGHT, and TL1A. We previously reported that DcR3 overexpressed in rheumatoid synovial fibroblasts (RA-FLS) stimulated by TNF $\alpha$ protects the cells from Fas-induced apoptosis. ${ }^{1}$ We recently reported that DcR3 binds to TL1A expressed on RA-FLS resulting in the negative regulation of cell proliferation induced by inflammatory cytokines. ${ }^{2}$ Further, we newly revealed the gene expression profiles in RA-FLS regulated by DcR3 by using microarray data analysis. ${ }^{3}$ Among the genes in the profile, we demonstrated the possible involvement of tryptophan hydroxylase 1 down-regulated, ${ }^{4}$ interleukin 12B up-regulated by DcR3 ${ }^{5}$ and centrosomal protein $70 \mathrm{kDa}^{6}$ in the pathogenesis of $\mathrm{RA}$. The profiles also indicated that Cadherin 2/type 1/N-cadherin $(\mathrm{CDH} 2)$ was up-regulated by DcR3 (fold change 1.93). ${ }^{3} \mathrm{CDH} 2$ has been reported to be associated with cell attachment and migration, ${ }^{7}$ osteoblast differentiation, ${ }^{8}$ and the proliferation of RA-FLS. ${ }^{9}$ The hemophilic interaction of $\mathrm{CDH} 2$ suppresses the proliferation of RA-FLS through increasing the P27 ${ }^{\mathrm{Kip} 1}$ that inhibit cell-cycle progression. ${ }^{9}$

Objectives: In this study, we analysed $\mathrm{CDH} 2$ expression in RA-FLS stimulated with $\mathrm{DcR} 3$ in detail to reveal the involvement of $\mathrm{CDH} 2$ and $\mathrm{DcR} 3$ in the pathogenesis of RA.

Methods : Real-time polymerase chain reaction (real-time PCR). RA-FLS were stimulated with various concentration of DcR3-Fc or $1,000 \mathrm{ng} / \mathrm{ml}$ of $\mathrm{lgG} 1$, or left untreated in serum-free Opti-MEM for 12 hour. The relative expression levels of $\mathrm{CDH} 2$ mRNA were quantified by real-time PCR.

Immunohistochemistry.

Anti-CDH2 antibody was applied to frozen sections of synovial tissues from patients with RA or OA for overnight. After that, the expression of $\mathrm{CDH} 2$ protein was evaluated by immunohistochemical analysis.

Results: Real-time PCR demonstrated that DcR3-Fc significantly increased the expression of CDH2 mRNA in RA-FLS $(104 \%$ with $10 \mathrm{ng} / \mathrm{ml}, 112 \%$ with $100 \mathrm{ng} /$ $\mathrm{ml}$, and $200 \%$ with $1000 \mathrm{ng} / \mathrm{ml}$ ). Immunohistochemistry revealed that $\mathrm{CDH} 2$ was expressed more in the sublining layer of rheumatoid synovium than OA synovium. Conclusions: In the gene expression profiles, we focused on $\mathrm{CDH} 2$ as the gene was highly up-regulated and belonged to major functional clustering categories; protein complex assembly, cell motility, regulation of transcription, cell membrane and glycosylation. In this study, we showed that the expression of $\mathrm{CDH} 2 \mathrm{mRNA}$ in RA-FLS was induced by DcR3 and that $\mathrm{CDH} 2$ was increased in the sublining layer of rheumatoid synovium. In this study, we demonstrated that DcR3 significantly induced $\mathrm{CDH} 2$ expression in RA-FLS. Considering the fact that $\mathrm{CDH} 2$ inhibits RA-FLS proliferation, DcR3 signalling may control the hyperplasia of RA synovium.

\section{REFERENCES:}

[1] Hayashi S, et al. Arthritis Rheum. 2007;56:1067-1074

[2] Takahashi M, et al. Int J Mol Med. 2011;28:423-427.

[3] Fukuda K, et al. Int J Mol Med. 2013;32:910-916.

[4] Maeda T, et al. Mol Med Rep. 2015;12:5191-5196.

[5] Fukuda K, et al., Mol Med Rep. 2016;13:3647-3652.

[6] Fukuda K, et al. Mod Rheumatol in press.

[7] Akitaya T, et al. Dev Dyn. 1992;194:12-20.

[8] Marie P. J Cell Physiol 2002;190:297-305.

[9] Nonomura Y, et al. J Rheumatol 2009;36:698-705.

Disclosure of Interest: None declared DOI: 10.1136/annrheumdis-2018-eular.2929

\section{$\mathrm{AB} 0066$}

COMBINATION OF IL-10 AND IL-18 BUT NOT IL-6 AND IL-18 INDUCES IFN-GAMMA PRODUCTION AND SURFACE EXPRESSION OF TRAIL ON NK CELLS

K. Sato, Y. Aizaki, H. Yazawa, T. Mimura. Department of Rheumatology and Applied Immunology, Saitama Medical University, Saitama, Japan

Background: Adult-onset Still's disease (AOSD) is a systemic inflammatory disease, the cause of which is largely unknown. AOSD has been recently classified as one of the autoinflammatory diseases, in which innate rather than acquired immunity plays an important role. Serum IL-18 has been shown to be significantly high in AOSD patients.

Objectives: The aim of this study was to quantify the levels of multiple cytokines in the serum of AOSD patients, and compare the serum cytokine profile with that of healthy controls. We also attempted to evaluate the effects of the cytokines that were upregulated in the AOSD serum on natural killer (NK) cells, since NK cells are cells of innate immunity and IL-18 has been shown to enhance their cytotoxicity. ${ }^{1}$

Methods: We quantified the serum levels of 10 cytokines (IFN- $\alpha$, IFN- $\gamma$, IL-1 $\beta$, IL2, IL-4, IL-6, IL-10, IL-12p70, IL-17A and TNF- $\alpha$ ) in patients with AOSD and healthy controls using multiplex bead array assays and IL-18 using ELISA. We next sorted NK cells from peripheral blood mononuclear cells (PBMCs) of healthy controls, stimulated them in vitro, and quantified the level of IFN- $\gamma$ in the culture supernatant by ELISA and also assessed the surface expression level of tumour necrosis factor-related apoptosis-inducing ligand (TRAIL) on the cells.

Results: Compared to samples from healthy controls, the mean serum levels of IL-6 and IL-18 from AOSD patients were significantly higher. IL-10 was detectable in some of the patients. Originally, IL-18 was identified as a stimulator of IFN- $\gamma$ production $^{2}$, however, serum IFN- $\gamma$ was below the detection limit. When NK cells were stimulated in vitro with IL-18 alone, the protein level of IFN- $\gamma$ in the culture supernatant was still below the detection limit. When we further added IL- 6 and/or $\mathrm{IL}-10$ to the culture, the combination of IL-10 and IL-18, but not IL- 6 and IL-18, induced IFN- $\gamma$. As IL- 6 is a classic pro-inflammatory cytokine and IL-10 is considered to be an anti-inflammatory cytokine, this result was rather unexpected. Thus, we evaluated the expression of IL-6 and IL-10 receptors on NK cells and found that IL-10 receptors (IL-10R and IL-10R $\beta$ ) were present, but IL- 6 receptors (IL-6R and gp130) were absent. We also assessed TRAIL expression on NK cells. Here, too, the combination of IL-10 and IL-18 induced TRAIL expression the most potently. Although a previous research showed that the combination of TLR3 and IL-18 signalling synergistically induced TRAIL on NK cells in an IFN- $\gamma$ dependent manner ${ }^{3}$, the addition of an anti-IFN- $\gamma$ antibody did not diminish the TRAIL expression.
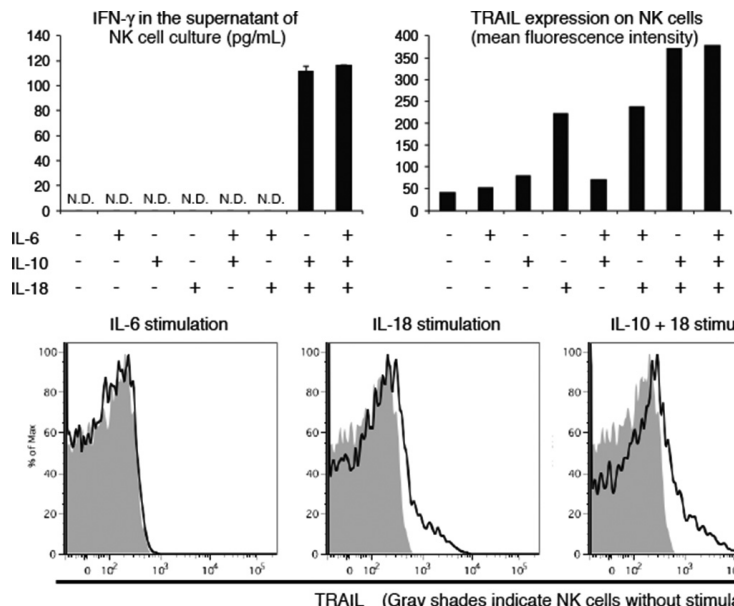

IL- $10+18$ stimulation

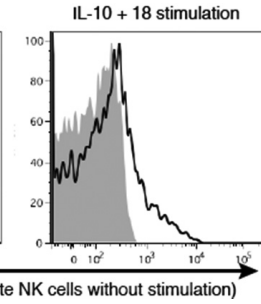

Abstract AB0066 - Figure 1

Conclusions: A combination IL-10 and IL-18, not IL-6 and IL-18, induced the production of IFN- $\gamma$ and surface expression of TRAIL in NK cells. This TRAIL expres sion did not evidently depend on IFN- $\gamma$. TRAIL was expected to be useful for the treatment of malignancy, but it turned out to be toxic to hepatocytes ${ }^{4}$. Since NK cells are rich in the liver and the abnormality of liver function is among the major symptoms in AOSD, we suggest that the combination of IL-10 and IL-18 may cause liver dysfunction by inducing TRAIL on NK cells in the liver.

\section{REFERENCES:}

[1] Akira S. Curr Opin Immunol. 2000;12:59-63.

[2] Okamura H, et al. Nature 1995;378:88-91.

[3] Tu Z, et al. Cell Immunol 2011;271:286-91.

[4] Jo M, et al. Nat Med. 2000;6:564-7. 